\title{
Performance Analysis of the Impact of the Propagation Environments on the WiMAX Service Classes
}

\author{
Yahya Abdullah Yahya Al zahrani \\ Researcher, College of Technology, Saudi Arabia \\ yalzahrani111@gmail.com
}

\begin{abstract}
Five different service classes are supported in mobile WiMAX IEEE 802.16 standard in order different needs of the mobile users to be fulfilled. In this paper we analyze the impact of the propagation environments on the WiMAX service classes and we measure the QoS performances of the service classes that are utilized from the mobile users. Simulation experiments are done utilizing the $n s-2.33$ simulator where all five service classes of the WiMAX are properly configured. Performances analysis of the service classes in mobile WiMAx is done measuring the average throughput, average delay and average jitter in various propagation environments. Obtained results give important conclusions about the QoS of the five service classes in mobile WiMAX in various propagation environments.
\end{abstract}

Keywords: delay, jitter, propagation environment, service class, throughput, WiMAX

\section{Introduction}

WiMAX technology (IEEE 802.16) is designed for wireless metropolitan area networks. The last version of this BWA (Broadband Wireless Access) system is already included in the

4G wireless network technology, recognized as $802.16 \mathrm{~m}$ mobile WiMAX standard. WiMAX networks can theoretically provide BWA up to 50 kilometers for stations that are fixed and 5 to 15 kilometers for mobile stations. This technology tolerates higher throughputs over longer distances and avoids interference at a minimum level.

Mobile WiMAX standard since the 802.16e version offers five classes of services in order to offer different QoS (Quality of Service) for various user requests. UGS (Unsolicited Grant Service), ertPS (extended real-time Polling Service) and rtPS (realtime Polling Service) are service classes that are used for real-time traffic amongst the users of the mobile WiMAX. On the other hand, nrtPS (non-real-time Polling Service) and BE (Best Effort) are service classes that are used for non-real-time traffic amongst the users. Each of these five service classes has specific QoS parameters defined with the bandwidth requirement and delay. Quality of Service (QoS) of the mobile WiMAX depends on the physical layer and medium access control (MAC) layer of the $802.16 \mathrm{~m}$, because these two layers arrange the base station and mobile station access. There are a lot of scientific papers related to QoS of the service classes in mobile WiMAX. Authors in [1] present a priority based upling scheduling scheme that maximizes the QoS performances of the real time service classes, especially ertPS service class. In [2] the authors improve the QoS performances of the WiMAX service classes, especially when the number of connections gets higher.

Nowadays, the study of the mobile WiMAX as a wireless broadband system, is great challenge for telecommunication service providers, because one of their main tasks is to perform analysis of the performances and cost related to the power consumption and required bandwidth. In this context, path loss propagation models are also very important 
area for researches, because the power of the signal is reduced in the real environments because of the path distance, free-space loss, reflection, scattering, diffraction, and absorption. Different kinds of environments, like urban, rural, suburban have great influence on the power of the signal. There are a lot of researches in this area in the recent years. In [3] the authors present a simulation study of various path loss empirical propagation models with measured field data in urban environment. Authors in [4] study the electromagnetic environment of WiMAX communication system working at 3.5 GHz. Radio signal loss is analyzed and compared in this work in urban, suburban and rural environment using different receiver antenna height. In [5] the authors present a channel model based on measurements conducted in commonly found scenarios in buildings, which include closed corridor, wide corridor and semi corridor.

The work presented in this paper includes thorough simulation results and discussion of the performances of the WiMAX service classes in different propagation environments. Many propagation environments with different parameters are taken into consideration and their impact on the average throughput, average delay and average jitter of all five service classes in mobile WiMAX is carefully analyzed. Important conclusions are provided from the simulation results that are of great benefit for researches in this area, as well as service providers of the mobile WiMAX technology.

The rest of the paper is organized as follows. Section 2 gives brief overview of the WiMAX service classes and propagation environments. In the Section 3 simulation environment that is used for the simulation results is presented. In Section 4 simulation scenarios and results are showed and explained. Finally, Section 5 concludes the paper.

\section{WiMAX Service Classes and Propagation Environments}

WiMAX technology in order to meet the different QoS requirements, like throughput (data rate), delay, jitter, packet loss, system availability, it uses various scheduling mechanisms to allocate uplink and downlink transmissions with the 5 service classes that are created for that purpose. QoS parameters of the all five service classes in mobile WiMAX are presented in Table 1.

\subsection{WiMAX Service Classes}

UGS (Unsolicited Grant Service) service class. This service class supports applications that generate periodically data packets with fixed size such is VoIP without silence suppression and T1/E1. Base station allocates for this service class fixed size data grants without receiving explicit requests from the subscriber stations (SS), in order to support the real-time requirements of this kind of applications and to reduce overhead by the bandwidth request-grant process.

rtPS (real-time Polling Service) service class. This service class is intended to support applications that generate in real-time variable size packets on a periodic basis like VoIP with silence suppression or MPEG video. For this service class base station permits subscriber stations to make periodic unicast requests and to define the size of the requested grant. There is a guarantee bandwidth request to be received on time by the SS, because the dedicate grant request is contention-free. Contention request opportunities cannot be used from the subscriber stations that are using this service class. 
Table 1. Mobile WiMAX Service Classes and QoS

\begin{tabular}{|c|c|c|}
\hline WiMAX service class & Applications & QoS Specification \\
\hline $\begin{array}{l}\text { UGS Unsolicited Grant } \\
\text { Service }\end{array}$ & VoIP & $\begin{array}{c}\text { Maximum Sustained Rate Maximum Latency } \\
\text { Tolerance } \\
\text { Jitter Tolerance }\end{array}$ \\
\hline $\begin{array}{c}\text { rtPS } \\
\text { real-time Polling Service }\end{array}$ & $\begin{array}{c}\text { Streaming } \\
\text { Audio or Video }\end{array}$ & $\begin{array}{c}\text { Minimum Reserved Rate Maximum Sustained } \\
\text { Rate Maximum Latency Tolerance } \\
\text { Traffic Priority }\end{array}$ \\
\hline $\begin{array}{l}\text { ertPS } \\
\text { extended real-time Polling } \\
\text { Service }\end{array}$ & $\begin{array}{l}\text { Voice with Activity Detection } \\
\text { (VoIP) }\end{array}$ & $\begin{array}{c}\text { Minimum Reserved Rate Maximum Sustained } \\
\text { Rate Maximum Latency Tolerance } \\
\text { Traffic Priority }\end{array}$ \\
\hline $\begin{array}{l}\text { nrtPS } \\
\text { non-real-Time Polling } \\
\text { Service }\end{array}$ & File Transfer Protocol (FTP) & $\begin{array}{l}\text { Minimum Reserved Rate Maximum Sustained } \\
\text { Rate Traffic Priority }\end{array}$ \\
\hline $\begin{array}{c}\text { BE } \\
\text { Best-Effort Service }\end{array}$ & Data Transfer, Web Browsing & $\begin{array}{l}\text { Maximum Sustained Rate } \\
\text { Traffic Priority }\end{array}$ \\
\hline
\end{tabular}

nrtPS (non real-time Polling Service). This service class is created to support applications in non-real time that need data grant bursts with variable size on a regular basis. So, this service class supports applications that are delay tolerant but might need higher data rates like FTP (File Transfer Protocol) applications. Similar to rtPS service class, base station permits subscriber stations to produce periodic unicast grant requests, although these requests are delivered at longer intervals. As a result of this, there is insurance here that even throughout congestion of the network, the subscriber stations will receive request opportunities. So, when using this class subscriber stations are also permitted to utilize contention request opportunities.

BE (Best Effort) service class. Applications that don't need any guarantee of QoS can be used with this service class, such as WWW (World Wide Web) access and telnet. Bandwidth request by this kind of applications is granted on space-available basis. Using this service class, subscriber stations can perform contention and contentionfree bandwidth requests. Still, when the system load is high contention-free bandwidth requests are not granted.

ertPS (extended real-time Polling Service). This service class is initiated in the same time when mobile WiMAX was introduced. It is a combination of the rtPS and UGS service classes. The latencies that originated from the bandwidth requests are removed, because the unsolicited unicast grants are provided by the base station. ertPS service class supports applications in real-time that generate data packets with variable size on a periodic basis, like voice with activity detection. 


\subsection{Propagation Environment and Models in NS-2}

Propagation environments are very important for the performance of the service classes in mobile WiMAX. For that purpose radio propagation models are implemented in various simulators for WiMAX. NS (network simulator) tools also implement radio propagation models that are used to predict the received signal power of each packet. In this paper ns-2.33 version of this simulator will be used for getting the simulations results. There is a receiving threshold at the physical layer of each wireless node designed in ns tool. If the signal power is below the receiving threshold when some packet is received, it is denoted as error and dropped by the MAC layer. Three propagation models exist in ns, free space model, two-ray ground reflection model and the shadowing model. Goal of this paper is fulfilled using the shadowing model of propagation.

Free space model and the two-ray ground reflection model of propagation envisage the received power as a deterministic function of distance. Communication range in both models is presented as an ideal circle. But, in real environment, the received power at some distance is a random variable because of the multi-path propagation effects, which is recognized as fading effects. Hence, the two mentioned models envisage the mean received power at distance. But, more universal and commonly-used model is well known as the shadowing model.

Shadowing model of propagation includes two parts. The first part is identified as path loss model, and it envisages the mean received power at some distance $d$ indicated by $P_{r}\left(d_{0}\right)$.

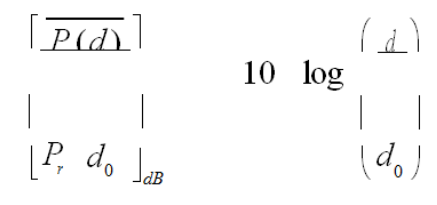

In equation (1) $\beta$ is called the path loss exponent that is empirically defined by field measurements. The second part of this shadowing model is related to the variation of the received power at some defined distance. This is performed with the log-normal random variable and it is of Gaussian distribution if it is measured in DB. Hence, taken as a whole, the shadowing model can be presented by the equation (2).

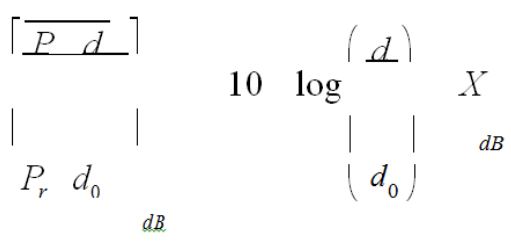

In equation (2) $X_{d B}$ is a Gaussian random variable with zero mean and standard deviation named sigma. Hence, the presented shadowing model expands the ideal circle model to more prosperous statistic model where nodes can only communicate probabilistically when they are located near the edge of the communication range. 
Table 2 presents the typical values of the $\beta$ path loss exponent that are set in different kinds of environments. Table 3 presents values of the standard deviation sigma that are set in different kinds of propagation environments using the shadowing model of propagation.

Table 2. Values of the $\beta$ path Loss Exponent in Different Environments

\begin{tabular}{|c|l|c|}
\hline \multicolumn{2}{|c|}{ Environment } & B path loss exponent \\
\hline \multirow{3}{*}{ Outdoor } & Free space & 2 \\
\cline { 2 - 3 } & Shadowed urban area & 2.7 to 5 \\
\hline \multirow{3}{*}{ In building } & Line-of-sight & 1.6 to 1.8 \\
\cline { 2 - 3 } & Obstructed & 4 to 6 \\
\hline
\end{tabular}

Table 3. Values of the Standard Deviation in Different Environments

\begin{tabular}{|l|c|}
\hline \multicolumn{1}{|c|}{ Environment } & B path loss exponent \\
\hline \multicolumn{1}{|c|}{ Outdoor } & 4 to 12 \\
\hline Office, hard partition & 7 \\
\hline Office, soft partition & 9.6 \\
\hline Factory, line-of-sight & 3 to 6 \\
\hline Factory, obstructed & 6.8 \\
\hline
\end{tabular}

\section{Simulation Environment}

Researches for this work are done using one of the best simulators for wireless networks, Network Simulator (NS). Simulations for this work are obtained using the version ns-2.33 of this simulator. There are several developed modules for mobile WiMAX available in the existing literature. But, the most adequate module that is focused on the MAC layer and its mechanisms for bandwidth allocation and QoS support is designed by the group of the University of Campinas [6]. This developed module is used as a basis for the researches conducted for this work. The topology for the simulation scenarios that were obtained includes a base station that is placed at the center of an area of 250x250 meters, where subscriber stations (SSs) are uniformly distributed around it. For each service class 5 subscriber stations are set, so there are 25 subscriber stations in total. Each of the subscriber stations has one uplink and one downlink flow which are mapped to the same service types. For the five service classes five types of corresponding traffic are utilized: voice, video, FTP, WEB and voice with silence suppression.

Exponential model with mean duration of 1.2 seconds for the on period and 1.8 seconds for the off period is used for the UGS service class. Packets with 66 bytes generated at 20 milliseconds are set for this service class. ertPS service class is simulated using the EVRC (Enhanced Variable Rate Codec) as a prototype for voice with silence suppression [7]. rtPS service class in the simulation scenarios is simulated utilizing real MPEG trace files. FTP traffic with exponential distribution with a mean of 512 kilobytes is used for generating the nrtPS service class. WEB traffic with hybrid Lognormal/Pareto distribution is used to generate the BE service class in the simulation scenarios. 
Table 4. Values of the Standard Deviation in Different Environments

\begin{tabular}{|l|c|c|}
\hline Propagation environment & Path loss exponent, $\boldsymbol{\beta}$ & Shadowing deviation in dB \\
\hline Outdoor, free space & 2 & 4 \\
\hline Outdoor, shadowed urban area & 5 & 6 \\
\hline $\begin{array}{l}\text { In building, line-of-sight, factory, } \\
\text { line-of-sight }\end{array}$ & 1.8 & 6.8 \\
\hline $\begin{array}{l}\text { In building, obstructed, factory, } \\
\text { obstructed }\end{array}$ & 6 & 7 \\
\hline $\begin{array}{l}\text { In building, obstructed, office, hard } \\
\text { partition }\end{array}$ & 6 & 9.6 \\
\hline $\begin{array}{l}\text { In building, obstructed, office, soft } \\
\text { partition }\end{array}$ & 6 & \multirow{2}{*}{6} \\
\hline
\end{tabular}

In this work 6 different simulation scenarios were created in order to generate the six different propagation environments of the shadowing propagation model, depicted in Table 4. The duration of the simulation scenarios is set to 100 seconds and each of the scenarios was generated 10 times with different seeds.

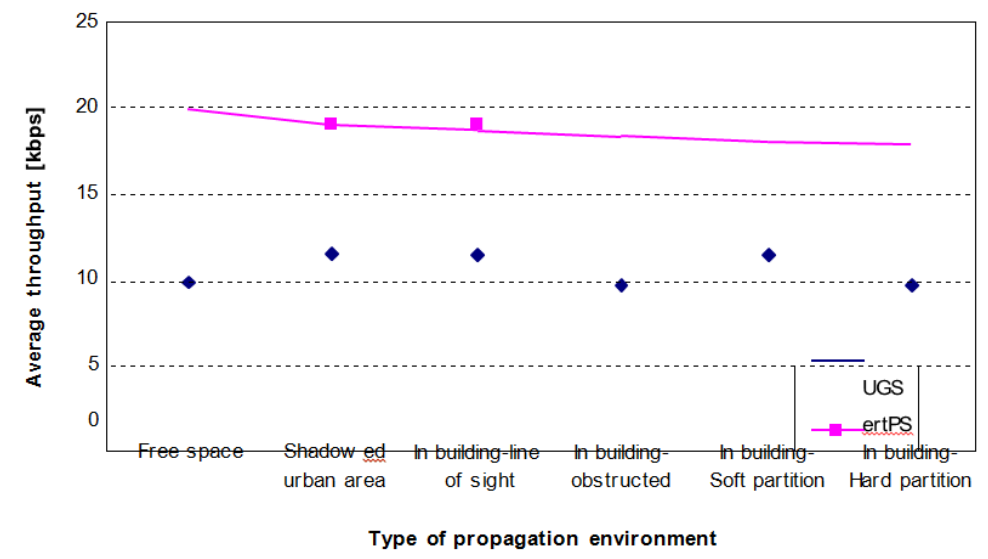

Figure 1. Average Throughput of UGS and ertPS Service Classes Expressed
in kbps

Results from the simulations are presented in this section. They are obtained after creating the simulation environment explained in the previous section. The simulation results are shown in average throughput, average delay and average jitter for all five service classes.

Figure 1 presents the average throughput of UGS and ertPS service classes that are used usually for voice traffic, as we mentioned above. We can notice that propagation environment has more impact on the ertPS service class, as the propagation environment is getting worse (free space, outdoor and indoor environment types) the average throughput of five SSs with ertPS is slowly decreasing under $20 \mathrm{kbps}$. UGS service class has the same average throughput of $9.84 \mathrm{kbps}$ in all shadowing model propagation types. This happens because the UGS service class is in the queue with high priority and because the load of the network is not so high in the simulated scenarios. 


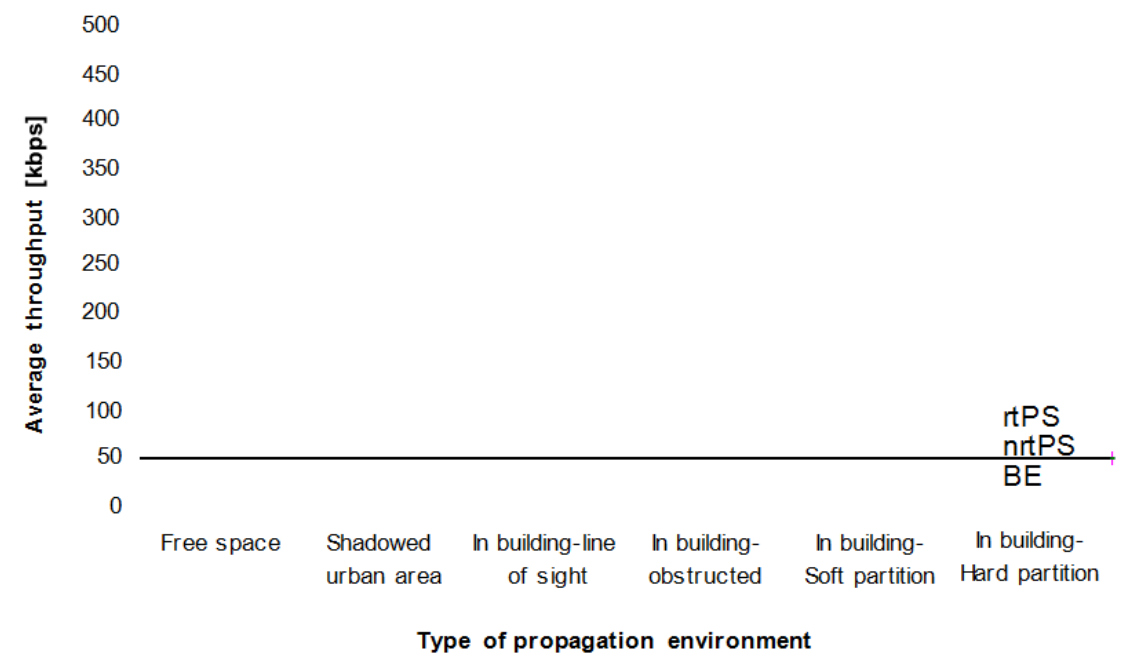

\section{Figure 2. Average Throughput of rtPS, nrtPS and BE Service Classes}

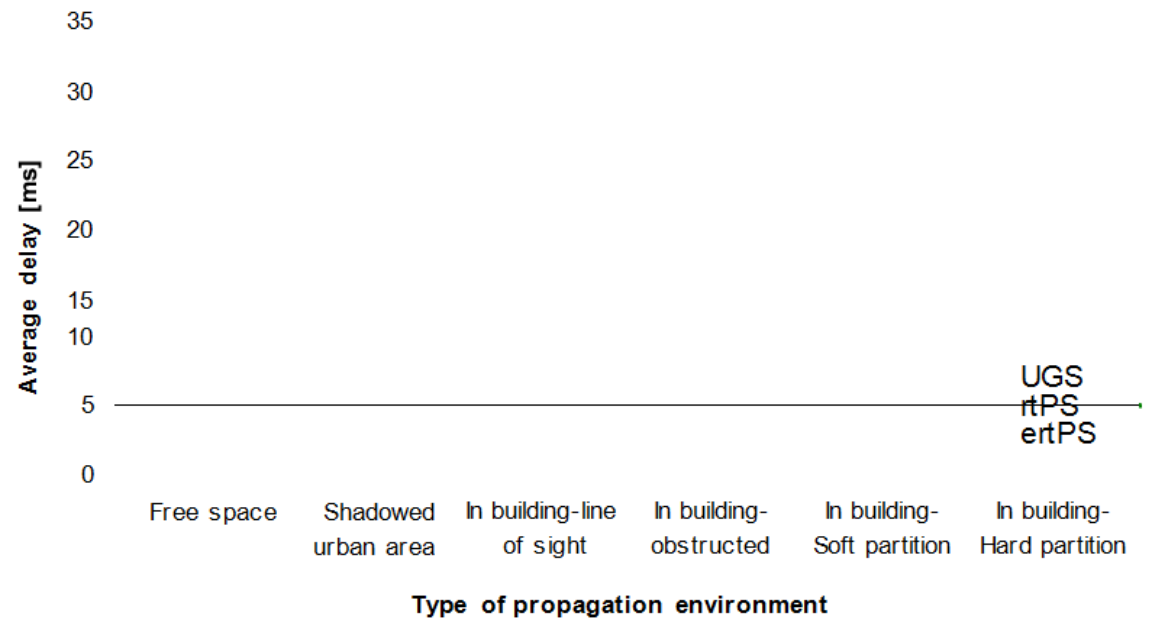

Figure 3. Average Delay of UGS, rtPS and ertPS Service Classes

Figure 2 shows the average throughput of rtPS, nrtPS and BE service classes. From this graph it can be concluded that shadowing propagation model types have more impact of non-real time service classes (nrtPS and BE). Average throughput of the real-time service class rtPS intended for video traffic is on the same level for all six propagation environments.

Figure 3 presents the average delay of the real-time service classes expressed in milliseconds. It is clearly shown that ertPS is the most sensitive service class to various types of shadowing propagation models. 


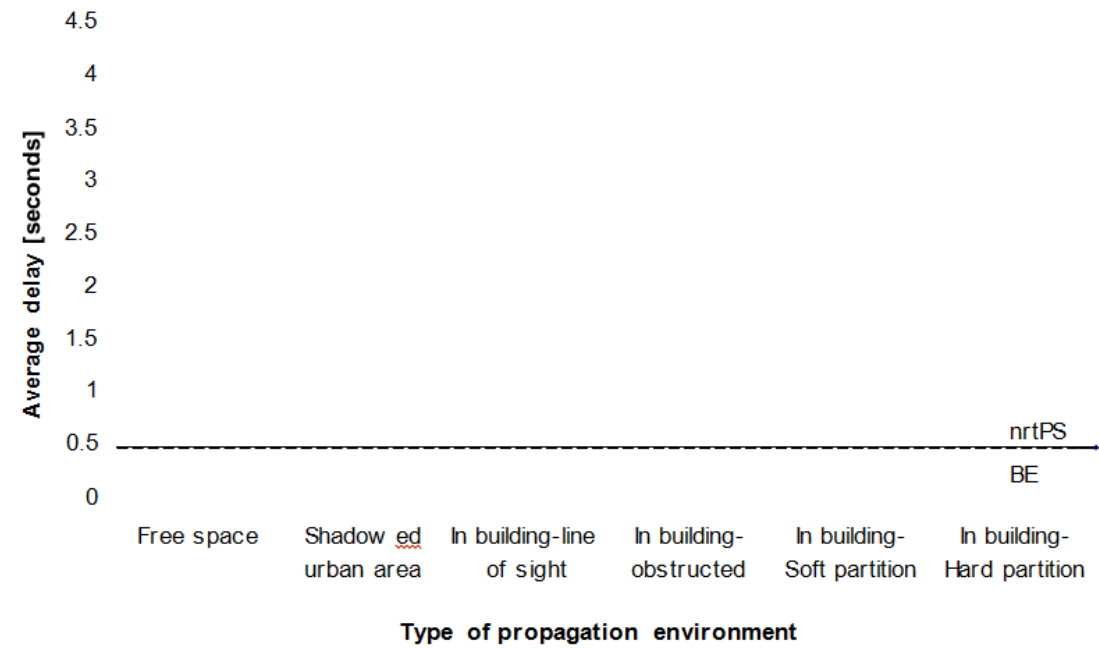

Figure 4. Average Delay of nrtPS and BE Service Classes Expressed in Seconds

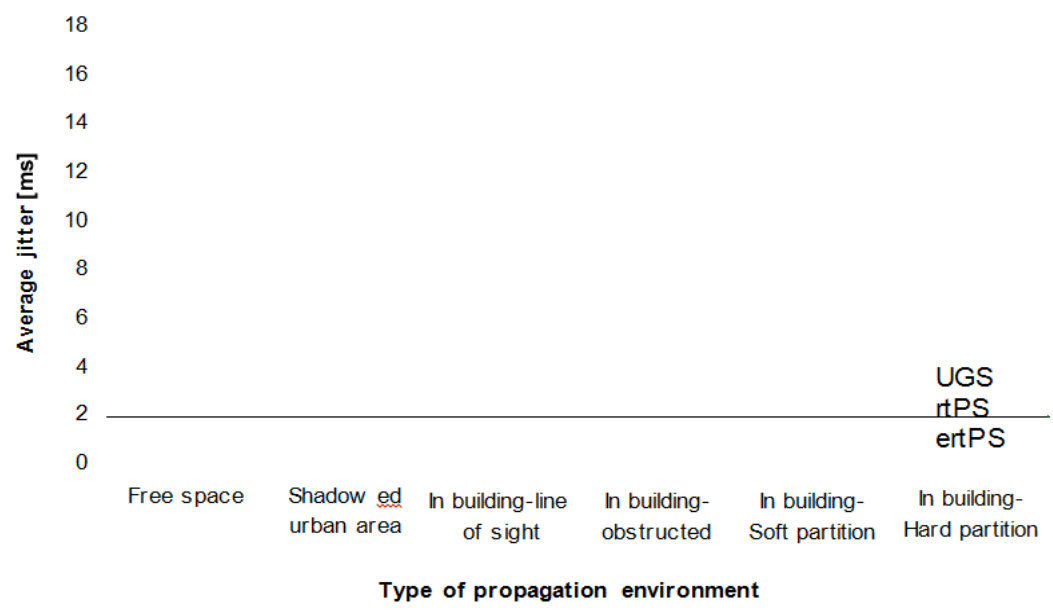

Figure 5. Average Jitter of UGS, rtPS and ertPS Service Classes

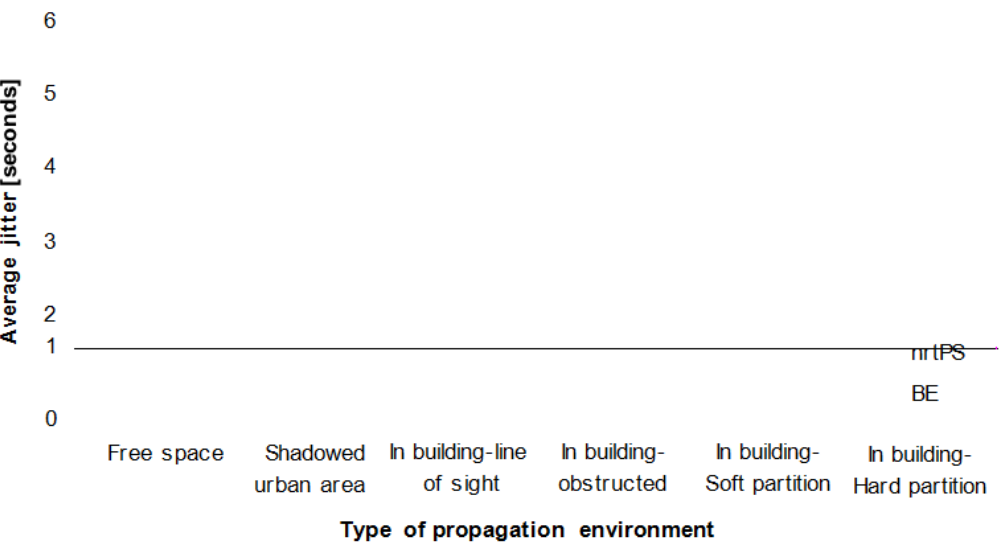

Figure 6. Average Jitter of nrtPS and BE Service Classes

Figure 5 and Figure 6 present the average delay results for all five service classes in different shadowing propagation model types. We can notice in Figure 5 that the 
worst average jitter from the real-time service classes has the rtPS service class. Similarly to results in average delay, different propagation environments have no impact on UGS service class. rtPS and ertPS service classes in Figure 5 have degraded average jitter when propagation environment changes from outdoor to indoor.

Figure 6 shows average jitter of the non-real time service classes, nrtPS and BE. It is clearly shown that for free space outdoor environment average jitter of nrtPS and $\mathrm{BE}$ is almost the same. But, as shadowing propagation models change from shadowed outdoor to indoor obstructed types, nrtPS service class degrades much faster than $\mathrm{BE}$ service class.

\title{
5. Conclusion
}

This paper presents analysis of the performances of all five service classes in mobile

WiMAX expressed in average throughput, average delay and average jitter. The researches are done utilizing the wimax module developed with the ns-2.33 simulator in linux environment. Simulation tests are conducted using a constant load of 5 subscriber stations for each of the five service classes (UGS, ertPS, rtPS, nrtPS and BE). Presents results in Section

4 show that service classes have different results for the six types of shadowing propagation

models. It is clearly proved that shadowing propagation model types have different influence on the service classes in mobile WiMAX.

Obtained simulation results in this paper are of great benefit for the science community as well as for the service providers, operators and administrators of the mobile WiMAX networks. These unique results can help a lot in more adequate treatment on different types of service classes of mobile WiMAX in different propagation environment, in order to have better QoS performances in practice.

\section{References}

[1] K. Jakimoski, T. Janevski, "Performance Analysis of the Real Time Service Classes in Mobile WiMAX", $10^{\text {th }}$ Conference for Informatics and Information Technology (CIIT 2013), (2013); Ohrid, Republic of Macedonia.

[2] K. Jakimoski and T. Janevski, "Improvement of the QoS for WiMAX Service Classes", TEM Journal, vol. 2, no. 2, (2013).

[3] S.S. Kale, and A.N. Jadhav, Performance Analysis of Empirical Propagation models for WiMAX in Urban Environment. IOSR Journal of Engineering, February (2013)

[4] H. Wadie Badri, S. Ghnimi, and A. Gharsallah,"Electromagnetic propagation environment effects on the WiMAX communication system", Mediterranean Microwave Symposium (MMS), (2011); Hammamet.

[5] B.T. Ahmed, J.L.M. Campos, and J.M.L. Mayordomo, "Propagation Path Loss and Materials Insertion Loss in Indoor Environment at WiMAX Band of 3.3 to $3.6 \mathrm{GHz}$ ", Wireless Personal Communications, vol. 66, no. 2, (2011), pp. 251-260.

[6] J.F. Borin, and N. da Fonseca, "Simulator for WiMAX Networks", Simulation Modelling Practice and Theory, vol. 16, (2008), pp. 817-833.

[7] 3GPP2 C.S0014-D, Version 3.0, Enhanced Variable Rate Codec (EVRC), (2010).

\section{Author}

\author{
Yahya Abdullah Yahya Al zahrani
}

Researcher, Kingdom of Saudi Arabia 
International Journal of Future Generation Communication and Networking Vol. 8, No. 1 (2015) 